\title{
Contactless Measurement of the Conductivity of Two-Dimensional Electrons in the Regime of Microwave-Induced Giant Magnetoresistance Oscillations
}

\author{
I. V. Andreev ${ }^{a}$, V. M. Murav'ev ${ }^{a, b}$, I. V. Kukushkinn ${ }^{a, b}$, J. H. Smet ${ }^{b}$, \\ K. von Klitzing ${ }^{b}$, and V. Umanskii ${ }^{c}$ \\ ${ }^{a}$ Institute of Solid State Physics, Russian Academy of Sciences, Chernogolovka, Moscow region, 142432 Russia \\ e-mail: andreev@issp.ac.ru \\ ${ }^{b}$ Max-Planck-Institut für Festkörperforschung, 70569 Stuttgart, Germany \\ ${ }^{c}$ Braun Center for Submicron Research, Weizmann Institute of Science, Rehovot, 76100 Israel
}

Received September 19, 2008

\begin{abstract}
Magnetic-field dependences of the conductivity of a two-dimensional electron system obtained by contact and contactless measurements in the regime of microwave-induced giant magnetoresistance oscillations have been comparatively analyzed. The contactless technique for studying the conductivity of two-dimensional electrons is based on measuring the attenuation of the RF signal propagating along a coplanar waveguide manufactured using lithography on the sample surface. It has been found that Shubnikov-de Haas oscillations of conductivity are observed in both techniques, whereas the microwave-induced giant magnetoresistance oscillations appear only in the contact measurements. This contradiction indicates that the contact and/or boundary regions of the two-dimensional system with a strong potential gradient play an important role for the observation of the induced magnetoresistance oscillations.
\end{abstract}

PACS numbers: 73.23.-b

DOI: $10.1134 / \mathrm{S} 0021364008210157$

In recent years, magnetotransport in high-mobility two-dimensional electron systems (2DES) under microwave irradiation has attracted considerable attention. To a great extent, this attention is caused by the recent discovery of microwave-induced giant magnetoresistance oscillations $[1,2]$ and zero-conductivity states $[3,4]$ that appear in the oscillation minima. The oscillations are $1 / B$ periodic and the positions of their maxima approximately correspond to the cyclotron resonance harmonics

$$
\omega=j \omega_{c}
$$

where $\omega$ is the frequency of the incident microwave radiation, $\omega_{\mathrm{c}}$ is the cyclotron frequency, and $j=1,2,3, \ldots$ is an integer. In the time that has passed since their discovery, the induced magnetoresistance oscillations were observed in contact transport measurements in the samples of different geometries: Hall bars [1, 3, 5], Corbino disks $[6,7]$ and square samples by the van der Pauw technique [8]. In spite of numerous theoretical and experimental studies, the physical nature of the oscillations is not quite clear and the investigation has revealed a set of unusual phenomena. In particular, it was found that the magnetoresistance oscillations do not coincide with the features of the microwave absorption and reflection by the sample and, consequently, they cannot be explained by the simple heating of the two-dimensional system due to the resonance absorption of radiation [9]. The insensitivity of the photoinduced magnetoresistance oscillations to the polarization of the microwave radiation [10], the observation of a large number of harmonics typical for Asbel-Kaner cyclotron resonance in metals [11], and the absence of magnetoresistance oscillations in optical experiments [12] allow one to state that the nature of the oscillations is significantly different from the usual bulk cyclotron resonance in semiconductors. The above facts indicate that the contact and/or boundary regions of the twodimensional electron system play an important role in the formation of the oscillations. In this work, a contactless study of the microwave response of the 2DES resistance was performed to prove this assumption. The essence of the used technique is to measure the attenuation of the RF signal propagating along the coplanar waveguide patterned on the sample surface. The signal attenuation is determined by the parameters of the waveguide and the real part of the diagonal component $\sigma_{x x}$ of the 2DES conductivity tensor.

The studies were carried out on a $30-\mathrm{nm}$ GaAs/AlGaAs single quantum well located at a depth of $161 \mathrm{~nm}$ from the structure surface. The mobility of the charge carriers in the structures with the twodimensional electron density $n=2.8 \times 10^{11} \mathrm{~cm}^{-2}$ was $\mu=8 \times 10^{6}$ and $15 \times 10^{6} \mathrm{~cm}^{2} / \mathrm{V}$ s at $T=4.2$ and $1.5 \mathrm{~K}$, 
respectively. The sample simultaneously containing the Hall bar and the coplanar waveguide sputtered on the top of the mesa above the two-dimensional electron gas was manufactured from the $4 \times 4 \mathrm{~mm}$ structure by means of optical lithography. The distance between the potential contacts of the $100-\mu \mathrm{m}$-wide Hall bar was $200 \mu \mathrm{m}$. The coplanar waveguide [13] is a kind of stripline and consists of a narrow central metallic strip located between two wide ground planes patterned on the substrate, in our case, the GaAs/AlGaAs heterostructure (Fig. 1). The length of the coplanar waveguide was $d=800 \mu \mathrm{m}$, the central strip width was $a=22 \mu \mathrm{m}$, and the distance between the side ground planes was $b=52 \mu \mathrm{m}$ to provide the characteristic impedance $Z_{0}=$ $50 \Omega$.

The sample was fixed at the end of a $7 \times 3.5-\mathrm{mm}$ rectangular waveguide and was immersed into the superconducting solenoid of a helium cryostat. The magnetic field was perpendicular to the sample plane. The sample was irradiated through the waveguide by microwave radiation in the frequency range from 40 to $80 \mathrm{GHz}$ with an incident power of no more than $100 \mathrm{~mW}$ at the channel input. During the transport measurements, an ac current of $1 \mu \mathrm{A}$ at a frequency of $40 \mathrm{~Hz}$ passed across the Hall bar with the lock-in detection of the resistance between the potential contacts. In the contactless measurements, the coplanar waveguide was connected by $50-\Omega$ coaxial cables between the $f_{0}=$ 0-2000 MHz RF source and the Schottky-diode detector placed outside the cryostat. The $10-\mu \mathrm{W}$ source power was modulated at a frequency of $210 \mathrm{~Hz}$ with the subsequent lock-in detection of the signal at the detector.

The major power losses during the signal passage through the coplanar line are due to the heating of the 2DES by the electric field concentrated in the coplanar waveguide slots. In this case, the transmission coefficient of the coplanar waveguide is described by the expression [14]

$$
\operatorname{Tr}=\exp \left(-\frac{Z_{0} \operatorname{Re}\left(\sigma_{x x}\right) d}{w}\right),
$$

where $w=(b-a) / 2$ is the slot width between the central conductor and the side ground planes. The expression holds for the transverse sizes $a$ and $b$, which are small compared to the wavelength of the RF signal and the small distance between the 2DES and the coplanar waveguide patterned on the heterostructure surface. Thus, the 2DES conductivity $\sigma_{x x}$ may be found from the transmission coefficient of the coplanar stripline. However, this is insufficient for finding the 2DES resistance $\rho_{x x}$. The components of the conductivity and resistance tensors of the two-dimensional electron gas are known to be related as

$$
\sigma_{x x}=\frac{\rho_{x x}}{\rho_{x x}^{2}+\rho_{x y}^{2}}
$$

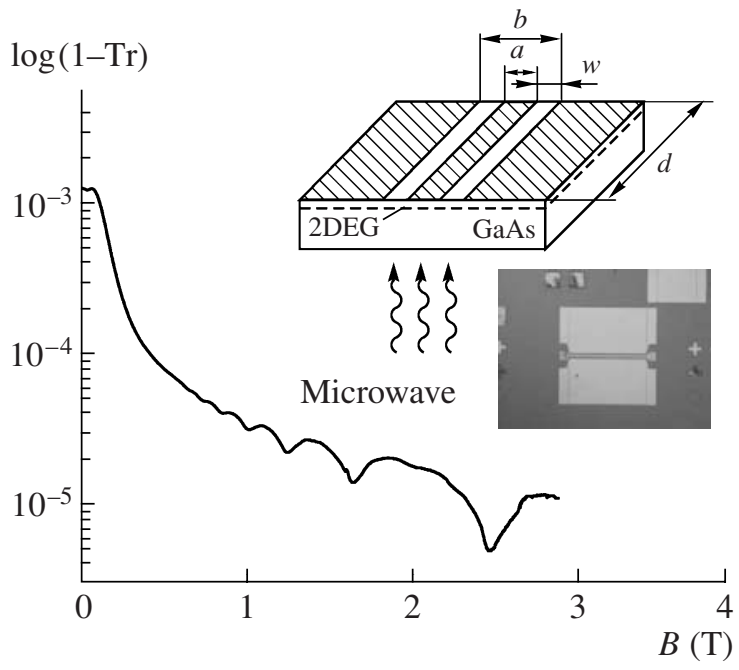

Fig. 1. Transmission of the coplanar waveguide at the frequency $f_{0}=700 \mathrm{MHz}$ versus the magnetic field in the absence of microwave irradiation of the sample at a temperature of $1.6 \mathrm{~K}$. The transmission coefficient Tr is normalized so that $\mathrm{Tr}=1$ corresponds to the damping-free propagation of the RF signal. The insets show the schematic and the photograph of the coplanar waveguide on the sample surface.

In the magnetic field range of interest, $\rho_{x y}^{2} \gg \rho_{x x}^{2}$; therefore,

$$
\rho_{x x} \approx-\rho_{x y}^{2} \frac{w \ln T r}{Z_{0} d} .
$$

Expression (4) will be used below to calculate $\rho_{x x}$ for the contactless measurements.

We tested the contactless technique by measuring Shubnikov-de Haas oscillations in the absence of microwave radiation. Figure 1 presents the magnetic field dependence of the transmission coefficient $\operatorname{Tr}$ of the coplanar waveguide at the RF signal frequency $f_{0}=$ $700 \mathrm{MHz}$. As follows from Fig. 2a, the experimental $\rho_{x x}(B)$ dependence that is obtained by the standard contact transport technique and is shown by the lower curve agrees well with the results of the contactless measurements shown by the upper curve. The quantitative difference between these two measurements is explained by the inaccurate description of the transmission of the coplanar waveguide by Eq. (2) and the electron localization effects [14] in the contactless measurements. The localization length decreases sharply with an increase in the signal frequency, which leads to a decrease in the mobility gap between the Landau levels. Therefore, the high-number Landau levels become unresolved with an increase in frequency. It should be mentioned that the contactless technique allows the resolution of the spin features of $\rho_{x x}$. Figure $2 \mathrm{~b}$ shows typical magnetoresistance $\rho_{x x}(B)$ curves under the continuous microwave irradiation at the frequency $f=$ $79 \mathrm{GHz}$. The curves were obtained with the use of the 

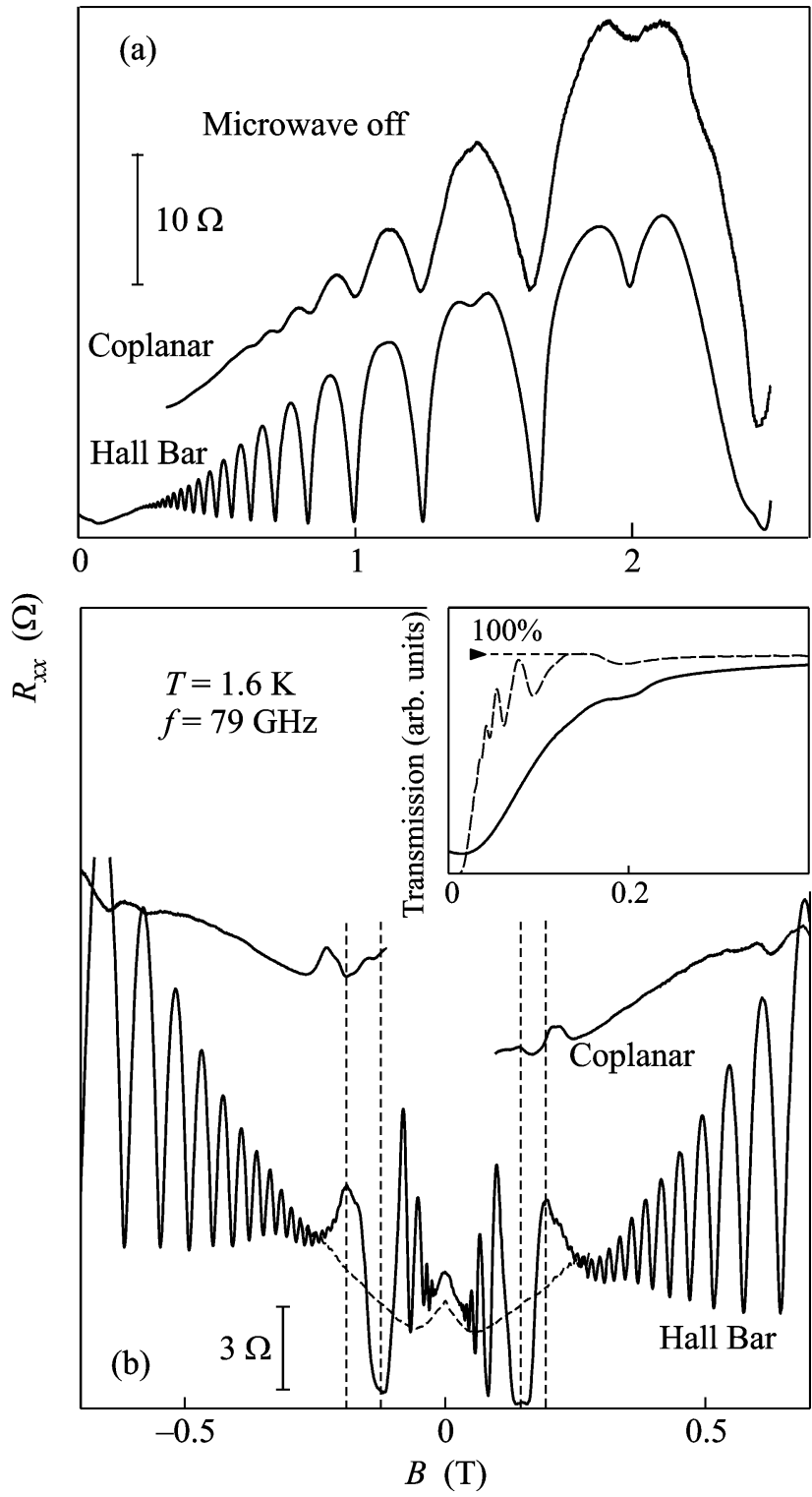

Fig. 2. (a) Magnetic-field dependences of the 2DES resistance in the absence of microwave irradiation obtained by the (lower curve) contact and (upper curve) contactless techniques. (b) The magnetic-field dependences of the 2DES magnetoresistance under the microwave irradiation obtained by the (lower solid curve) contact and (upper solid curve) contactless techniques. The 2DES magnetoresistance in the absence of microwave irradiation is shown by the dashed line for comparison. The inset shows the transmission coefficient of the coplanar waveguide (solid line) measured in the experiment and (dashed line) calculated with the use of the results of contact measurements.

standard transport technique. The dashed line shows the magnetoresistance signal without microwave irradiation. Clearly, the well-studied $1 / B$ oscillations and zeroresistance states are observed at small magnetic fields. However, the $\rho_{x x}$ curve in Fig. $2 \mathrm{~b}$ obtained by means of the contactless technique displays only minor features at the magnetic fields that do not correspond to the
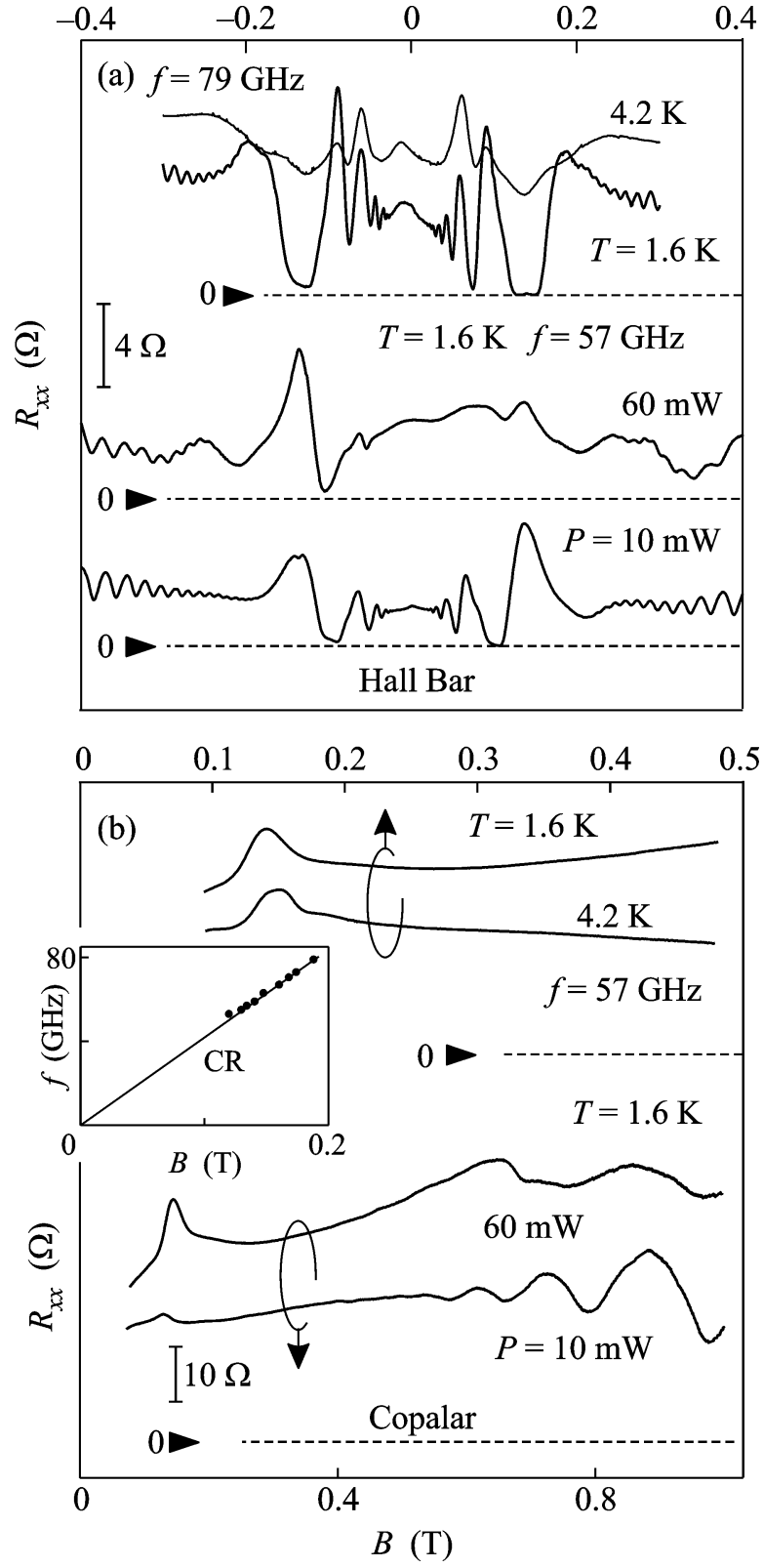

Fig. 3. Magnetic-field dependences of the 2DES resistance obtained by the (a) contact and (b) contactless techniques under the microwave irradiation (upper curves) for the two temperatures of the sample, $T=4.2$ and $1.6 \mathrm{~K}$ and (lower curves) two values of the microwave power, $P=10$ and $60 \mathrm{~mW}$. The inset shows the magnetic dispersion of the observed plasma mode.

extrema of the transport magnetoresistance oscillations. Below, we show that the peaks in the contactless magnetoresistance signal are associated with the heating of the 2DES due to the excitation of bulk magnetoplasma modes. The transmission of the coplanar stripline measured under the continuous microwave irradiation of the 2DES at the frequency $f_{0}=700 \mathrm{MHz}$ is presented in the inset in Fig. 2a by the solid line. The finite transmission of the line at zero magnetic field is 
caused by the direct power transmission between the input and output coaxial cable. The minimum associated with the magnetoplasma resonance is clearly seen in the transmission signal. The dashed line shows the transmission signal obtained from the $\rho_{x x}(B)$ and $\rho_{x y}(B)$ curves according to Eq. (2). A considerable difference between the two transmission curves is seen at small magnetic fields.

To test the magnetoplasma origin of the magnetoresistance peaks observed in the contactless measurements, we studied the behavior of the peaks with the variation of the 2DES temperature and microwave power. Figure $3 a$ presents the $\rho_{x x}$ curves obtained with the use of the transport technique at the temperatures $T=1.6$ and $4.2 \mathrm{~K}$ under continuous microwave irradiation at the frequency $f=79 \mathrm{GHz}$. The figure also presents two curves obtained at $T=1.6 \mathrm{~K}, f=57 \mathrm{GHz}$, and microwave powers $P=10$ and $60 \mathrm{~mW}$. Obviously, an increase in both the temperature from 1.6 to $4.2 \mathrm{~K}$ and the microwave power destroys the zero-resistance states. The induced magnetoresistance oscillations also exhibit substantial distortions: their amplitude decreases and at most two magnetoresistance peaks can be resolved, whereas five distinct photoinduced peaks are observed at $T=1.6 \mathrm{~K}$ and $P=10 \mathrm{~mW}$. At the same time, as shown in Fig. 3b, the features observed in the contactless measurements remain almost unchanged with temperature and are considerably amplified with an increase in the microwave power. This behavior clearly indicates that the peaks seen in the contactless measurements cannot be associated with the induced magnetoresistance oscillations, but, rather, have the magnetoplasma origin. The magnetic field dependence of the peak position at different microwave frequencies is shown in the inset in Fig. 3b. In high magnetic fields, the magnetoplasma mode nearly coincides with the cyclotron resonance, because the plasma frequency of the screened plasmon with a wave vector corresponding to the structure size is much lower than the cyclotron frequency [12].

To conclude, the contactless technique for studying the photoinduced effects in the magnetoresistance of 2DES is developed. It is shown with the use of the technique that the photoinduced magnetoresistance oscilla- tions observed in the transport measurements are not seen in the contactless experiments. The experimental results indicate that the contact and/or boundary regions of the 2DES with a strong field gradient play an important role in the origin of photoinduced magnetoresistance oscillations.

This work was supported by the Russian Foundation for Basic Research, Deutsche Forschungsgemeinschaft, INTAS, and the Russian Federal Agency for Science and Innovations.

\section{REFERENCES}

1. M. A. Zudov, R. R. Du, J. A. Simmons, et al., Phys. Rev. B 64, 201311 (2001).

2. P. D. Ye, L. W. Engel, D. C. Tsui, et al., Appl. Phys. Lett. 79, 2193 (2001).

3. R. G. Mani, J. H. Smet, K. von Klitzing, et al., Nature 420, 646 (2002).

4. M. A. Zudov, R. R. Du, L. N. Pfeiffer, et al., Phys. Rev. Lett. 90, 046807 (2003).

5. S. I. Dorozhkin, J. H. Smet, V. Umansky, et al., Phys. Rev. B 71, 201306 (2005).

6. C. L. Yang, M. A. Zudov, T. A. Knuuttila, et al., Phys. Rev. Lett. 91, 096803 (2003).

7. A. A. Bykov, Pis'ma Zh. Éksp. Teor. Fiz. 87, 638 (2008) [JETP Lett. 87, 551 (2008)].

8. A. A. Bykov, D. R. Islamov, A. V. Goran, et al., Pis'ma Zh. Éksp. Teor. Fiz. 86, 891 (2007) [JETP Lett. 86, 779 (2007)].

9. S. A. Studenikin, M. Potemski, A. S. Sachrajda, et al., arXiv:cond-mat/0404411v2 (2004).

10. J. H. Smet, B. Gorshunov, C. Jiang, et al., Phys. Rev. Lett. 95, 116804 (2005).

11. M. Ya. Azbel and E. A. Kaner, Zh. Éksp. Teor. Fiz. 32, 896 (1956) [Sov. Phys. JETP 5, 730 (1956)].

12. I. V. Kukushkin, V. M. Muravev, J. H. Smet, et al., Phys. Rev. B 73, 113310 (2006).

13. C. P. Wen, IEEE Trans. Microwave Theory and Appl. 17, 1087 (1969).

14. L. W. Engel, D. Shahar, C. Kurdak, et al., Phys. Rev. Lett. 71, 2638 (1993).

Translated by A. Safonov

JETP LETTERS Vol. $88 \quad$ No. 92008 